\title{
Experimental and numerical evaluation of interdigitated electrode array for monitoring gaseous sterilization processes
}

\author{
Jan Oberländer ${ }^{1,2}$, Zaid B. Jildeh ${ }^{1}$, Patrick Kirchner ${ }^{1}$, Luisa Wendeler ${ }^{1}$, Alexander Bromm ${ }^{1}$, Heiko Iken $^{1,2}$, \\ Patrick Wagner, Michael Keusgen ${ }^{4}$ and Michael J. Schöning ${ }^{1,2}$ \\ ${ }^{1}$ Institute of Nano- and Biotechnologies (INB), FH Aachen Campus Jülich, Jülich, Germany \\ ${ }^{2}$ Peter-Grünberg Institute (PGI-8), Forschungszentrum Jülich GmbH, Jülich, Germany \\ ${ }^{3}$ Soft Matter and Biophysics, Catholic University Leuven, Leuven, Belgium \\ ${ }^{4}$ Institute of Pharmaceutical Chemistry, Philipps-University Marburg, Marburg, Germany
}

Contact: oberlaender@fh-aachen.de

\begin{abstract}
A sensor to evaluate sterilization processes with hydrogen peroxide vapor has been introduced. Experimental, analytical and numerical methods have been applied to evaluate and study the sensor behavior. The sensor set-up is based on a planar interdigitated electrode array of a total sensing area of $20 \mathrm{~mm}^{2}$. Sensor measurements with and without microbiological spores have been conducted. Further measurements have been performed after chemical sterilization using vaporized hydrogen peroxide. The measurements were verified using a predefined published equation used to describe periodic planar interdigitated structures. Additionally, a two-dimensional periodic finite element method model was designed and simulated to validate the experimental findings.
\end{abstract}

Keywords: sterilization process; hydrogen peroxide; interdigitated electrodes (IDE); FEM model

\section{Introduction}

To increase the hygienic level of materials and equipment used in pharmaceutical, medical and packaging industry, a wide range of sterilization processes and media are applied. All sterilization processes serve a common goal of eliminating harmful bacteria and their durable form (spores) located on the surface to be sterilized. Based on the nature of the surface, different sterilization procedures (single or multi-step processes) are applied. These include the sterilization by heat (dry or wet), irradiation (e.g., UV, gamma or electron beam) or even chemicals (e.g., hydrogen peroxide $\left(\mathrm{H}_{2} \mathrm{O}_{2}\right)$ or peracetic acid). Chemical sterilization by $\mathrm{H}_{2} \mathrm{O}_{2}$ has gained favor in the food packaging industry [1]. Here, one variation of the process includes the treatment of pre-heated multilaminated packages holding -for example- milk or juice. In this process, an air $-\mathrm{H}_{2} \mathrm{O}_{2}$ mixture is vaporized at temperatures up to $300{ }^{\circ} \mathrm{C}$ and guided into the pre-heated packages. The microbicidal and sporicidal properties of the $\mathrm{H}_{2} \mathrm{O}_{2}$ can be attributed to the radical formation during thermal decomposition at elevated temperatures [1]. Furthermore, vaporized $\mathrm{H}_{2} \mathrm{O}_{2}$ possesses an advantage over other chemical sterilization media; it decomposes to environmental friendly end-products: water and oxygen.

Monitoring the sterilization efficiency is usually performed by time-consuming and lab-intensive microbiological challenge tests (e.g., countreduction test, end-point test). For these sterilization tests, resilient microorganisms to the sterilization process are immobilized on the surface of test packages. Hence, to evaluate the sterilization efficiency of $\mathrm{H}_{2} \mathrm{O}_{2}$ vapor spores of Bacillus (B.) atrophaeus are typically applied as a bio-indicator [2].

Interdigitated electrode (IDE) arrays are common devices used in monitoring biological or chemical reactions, either in liquid or in gas phase [3-5]. IDE arrays applied as gas sensors are most often used in combination with polymers or metal oxides as transducer materials.

In this work, a time and resource efficient monitoring system based on an IDE sensor setup will be presented. For the present sensor, the IDE structure has been adapted to monitor alterations on spores of $B$. atrophaeus. To achieve that goal, the spores must be immobilized first on the surface of the IDE sensor from an ethanol-based spore suspension by evaporating it at room temperature, as applied for the industrial challenge tests.

Experimental data are achieved using impedimetric analysis of immobilized IDE structure before and after sterilization. This follows a verification and validation process of experimental findings utilizing pre-developed analytical expressions and evaluation with the help of numerical finite element methods (FEM) models, respectively. 


\section{Materials and methods}

\section{Sensor fabrication}

The fabrication of the IDE arrays has been previously described in [6]. However, for this work, a glass substrate has been applied instead of the previous selection of a $\mathrm{Si} / \mathrm{SiO}_{2}$ substrate. A schematic of the sensor processing steps is shown in Fig. 1, where the fabrication starts with a cleaning of the glass wafer with acetone followed by isopropanol and deionized water. Later, a photoresist layer of AZ5214E is spin-coated to the glass surface and patterned by UV light exposure. Vapor deposition of titanium (Ti) and platinum $(\mathrm{Pt})$ is performed after photoresist development. The thicknesses of the layers are 10 and $100 \mathrm{~nm}$, respectively. Afterwards, a lift-off process in DMSO was conducted, enhanced with an ultrasonic bath, leaving the IDE structure as shown in Fig. 1 (f). A further lithography process (based on SU-8) is applied to form the sensor cavity required to hold the spores of $B$. atrophaeus spores. The spore sample is applied from an ethanol-based suspension $(10 \mu \mathrm{l})$, resulting in a spore concentration of $10^{6}$ colony forming units (cfu) per IDE. a)

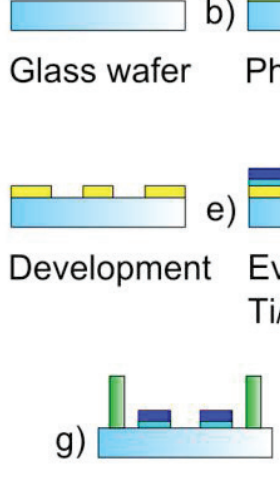

SU-8 walls h)

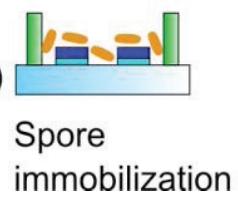

Fig. 1: Schematic representation of the sensor fabrication steps.

The resulting sensor chip from the fabrication process is presented in Fig. 2. Here, a differential set-up of two IDEs (glass substrate base; finger width and interspacing: $5 \mu \mathrm{m}$; finger length: $3.25 \mathrm{~mm}$; number of fingers: 614) represents a single sensor assembly. One IDE structure serves as a reference element, while on the second structure microbiological spores of $B$. atrophaeus are immobilized. Later, the sensor assembly is subjected to the sterilization process, which induces distinct alterations to the microbiological spores. These changes result in an impedance/capacitance change of the IDEs that can be quantified using an impedance analyzer.

\section{Impedimetric characterization}

The electrical characterizations of the IDE arrays are performed on a point-probe station in combination with an Agilent E4980A impedance analyzer. An alternating voltage of $20 \mathrm{mV}, 0 \mathrm{~V}$ bias voltage, was applied between the electrode terminals. The resulting current is used to determine the impedance and phase of each sensor. A set of two IDEs is utilized to evaluate the sterilization process. On one of the IDEs microbiological spores of $B$. atrophaeus are immobilized. The second IDE is left blank to serve as a reference.

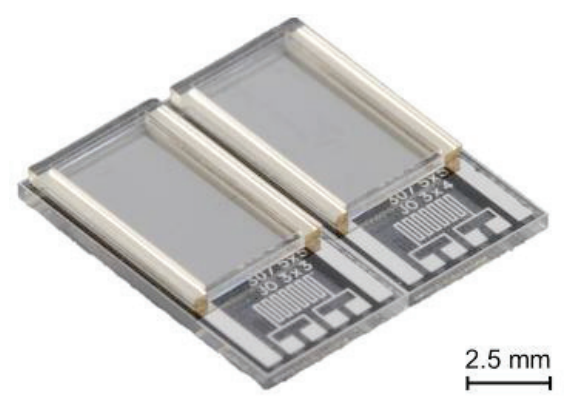

Fig. 2: Differential set-up of two IDE arrays with two independent sensing areas of $20 \mathrm{~mm}^{2}$.

The sensors as well as microbiological samples are sterilized on a predesigned test-rig that is a representative of the sterilization module used in an industrial aseptic filling machine [7]. The $\mathrm{H}_{2} \mathrm{O}_{2}$ vapor is generated from an aqueous $\mathrm{H}_{2} \mathrm{O}_{2}$ solution $(35 \% \mathrm{w} / \mathrm{w})$ and is fed into an air stream as carrier gas. The air $-\mathrm{H}_{2} \mathrm{O}_{2}$ mixture is vaporized at high temperature and is then guided into the sterilization chamber. The sensors and microbiological samples are exposed for $0.3 \mathrm{~s}$ into the vapor stream. The exposure time has been evaluated in pre-studies, whereby full sterility was proven at an $\mathrm{H}_{2} \mathrm{O}_{2}$ concentration of $7.5 \% \mathrm{v} / \mathrm{v}$, gas temperature of $240{ }^{\circ} \mathrm{C}$ and an air volume flow of $10 \mathrm{~m}^{3} / \mathrm{h}$ [8].

\section{Analytical verification}

Analytical expressions to facilitate theoretical descriptions of IDE arrays can be found in literature [9-11]. The fringing field between the electrodes of an IDE can be described using a first-order elliptical integral, as described in [11]. In order to include the transverse field between the electrode fingers, an extension and further enhancement to the equation was introduced by $[9,10]$. 


$$
C=L(N-1)\left(\frac{\varepsilon_{0} \varepsilon_{r, t}}{2} \frac{K\left(\left(1-k^{2}\right)^{1 / 2}\right)}{K(k)}+2 \varepsilon_{0} \varepsilon_{r, m} \frac{t}{s}\right)
$$

In eq. (1), $L$ represents the finger length, $N$ number of fingers, $\varepsilon_{0}$ the dielectric permittivity of vacuum, $\varepsilon_{r, t}$ the total relative permittivity surrounding the electrodes (empty sensor: air and glass; sensor with spores: porous spore layer and glass). $K(k)$ is the elliptic integral of first order, representing the fringing field. The modulus $k$ given in eq. (2) is specified by the periodic electrode structure, $s, w$ and $t$ are the interspacing between the fingers, width of the electrode fingers and electrode thickness, respectively. $\varepsilon_{r, m}$ describes the relative permittivity present between the electrodes representing the transverse field (empty sensor: air; sensor with spores: porous spore layer).

$$
k=\cos \left(\frac{\pi}{2} \frac{w}{s+w}\right)
$$

\section{Numerical validation}

Simulation of the full three-dimensional (3D) IDE structure is possible using a geometric simplification procedure. In which the redundancy in the finger design allows the use of numerical periodic boundary conditions. In addition, the depth of the finger structure can also be seen as an extension parameter of a two-dimension (2D) model. Hence, with the decrease in geometric order, thorough analysis was possible to include the distribution of the spore's layer. The IDE sensor structure used for the numerical simulation is shown in Fig. 3.

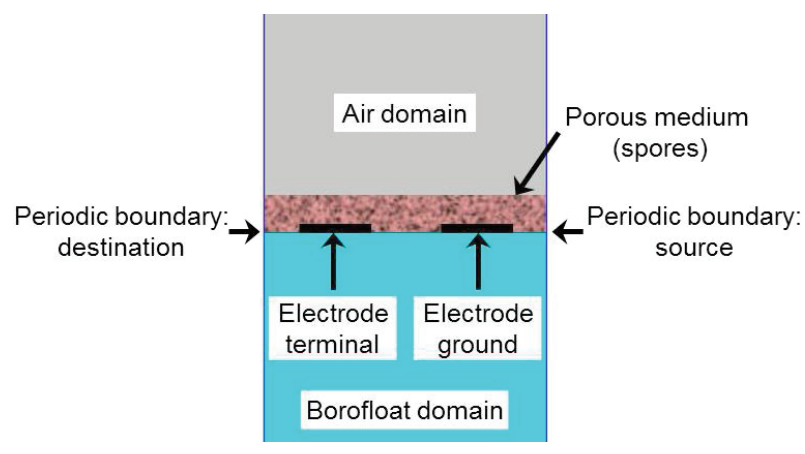

Fig. 3: Schematic of the sensor structure used for numerical simulation.

The model of the sensor includes an electrode finger pair situated on top of a glass substrate, which is exposed to air. The porous medium surrounding the finger pair represents the immobilized spores. The FEM-based numerical model solves Gauss's law of electric field distribution using the defined value of electric potential $\left(U_{0}: 20 \mathrm{mV}\right)$ as the dependent variable. Thereby, the capacitance of the design can be calculated by FEM using the surface integral $(\Omega)$ of the stored electric energy $W_{e}$ :

$$
C=N \frac{2}{U_{0}^{2}} \int_{\Omega} W_{e} d \Omega
$$

\section{Results and discussions}

\section{Results of impedimetric characterization}

The impedimetric characterizations were carried out in air and after the drying process of the ethanol-based spore suspension. Fig. 4 shows the average resulting Bode plots of four independent sensors. The plot of the bare sensor structure (black lines) exhibits a pure capacitive behavior (phase angle at $-90^{\circ}$ ) within the studied frequency range $(200 \mathrm{~Hz}$ to $1 \mathrm{MHz})$. The immobilization of spores results in a decrease of impedance value and change in phase angle (blue lines) which can be related to an enhancement of the conductivity between the electrode structures. The capacitive behavior of the sensor is predominant, especially at frequencies above $100 \mathrm{kHz}$.

After the sterilization process an alteration in impedance and phase can be observed (orange lines). The impedance is lower compared to the measurements with intact spores. This change can be related to previous investigations (refer to [8]), where a collapse and rupture of spores was described. A further increase in the conductivity might be related to the release of dipicolinic acid (DPA) after treatment by oxidizing agents [12].

To enable a total analysis and comparison between the different sensor states (bare structure, immobilized spores and after sterilization), the sensor's capacitance was calculated by an equivalent electric circuit analysis eq. (4).

$$
C=\frac{-\sin (\varphi)}{2 \pi f \cdot Z}
$$

Here, $\varphi$ represents the phase angle, $Z$ the impedance at frequency $f$.

The capacitance values at a frequency of $3 \mathrm{kHz}$ are depicted in Fig. 5. After the immobilization of the spores on the sensor surface, a capacitance increase of about $49.6 \mathrm{pF}$ was derived. The previously discussed impact of the sterilization process increases the capacitance by about $19.3 \mathrm{pF}$. The measurement errors arise from the statistical variations in the coverage of the sensors. Nonetheless, the capacitance change caused by the sterilization was not less than $15 \mathrm{pF}$ for all the four tested sensors. 


\section{Result of analytical verification}

The analytical expression introduced in the materials and methods section was applied to calculate the capacitance value of the bare IDE structure. By applying the geometric sensor parameters in eq. (1), a capacitance value of 58 pF was determined. Comparing this analytical value with the experimental result a relative error of $9.2 \%$ was derived, which can be attributed to uncertainties of the sensor fabrication, ambient temperature and humidity effects.
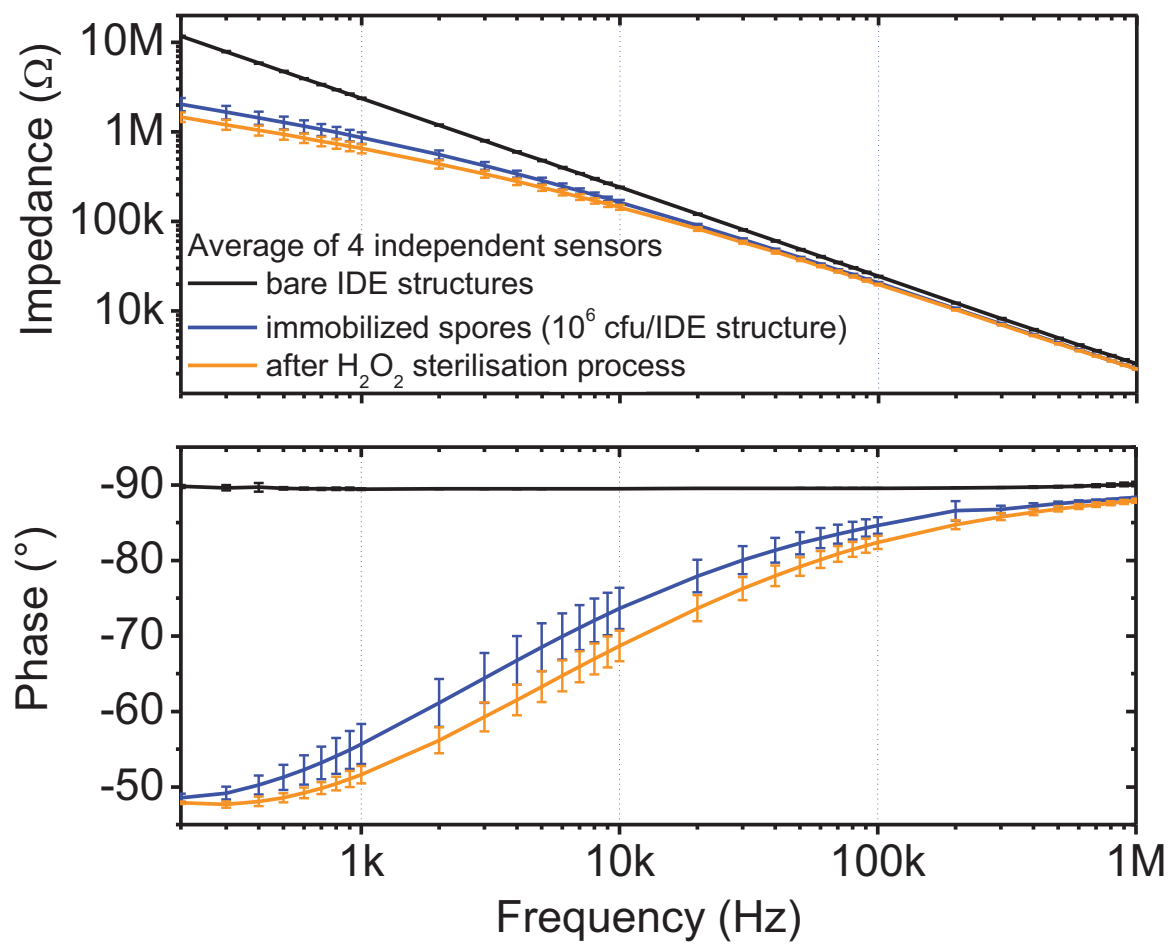

Fig. 4: Averaged Bode plot of four independent sensors. Characterizations in air: bare structure (black lines), after immobilization of $10^{6} \mathrm{cfu} / \mathrm{IDE}$ (blue lines) and after $\mathrm{H}_{2} \mathrm{O}_{2}$ vapor sterilization for $0.3 \mathrm{~s}$ at $\mathrm{c}\left(\mathrm{H}_{2} \mathrm{O}_{2}\right)$ :

$7.5 \% \mathrm{v} / \mathrm{v}, \mathrm{T}_{\text {gas }}: 240{ }^{\circ} \mathrm{C}, \dot{\mathrm{V}}_{\text {air }}: 10 \mathrm{~m}^{3} / \mathrm{h}$ (orange lines).

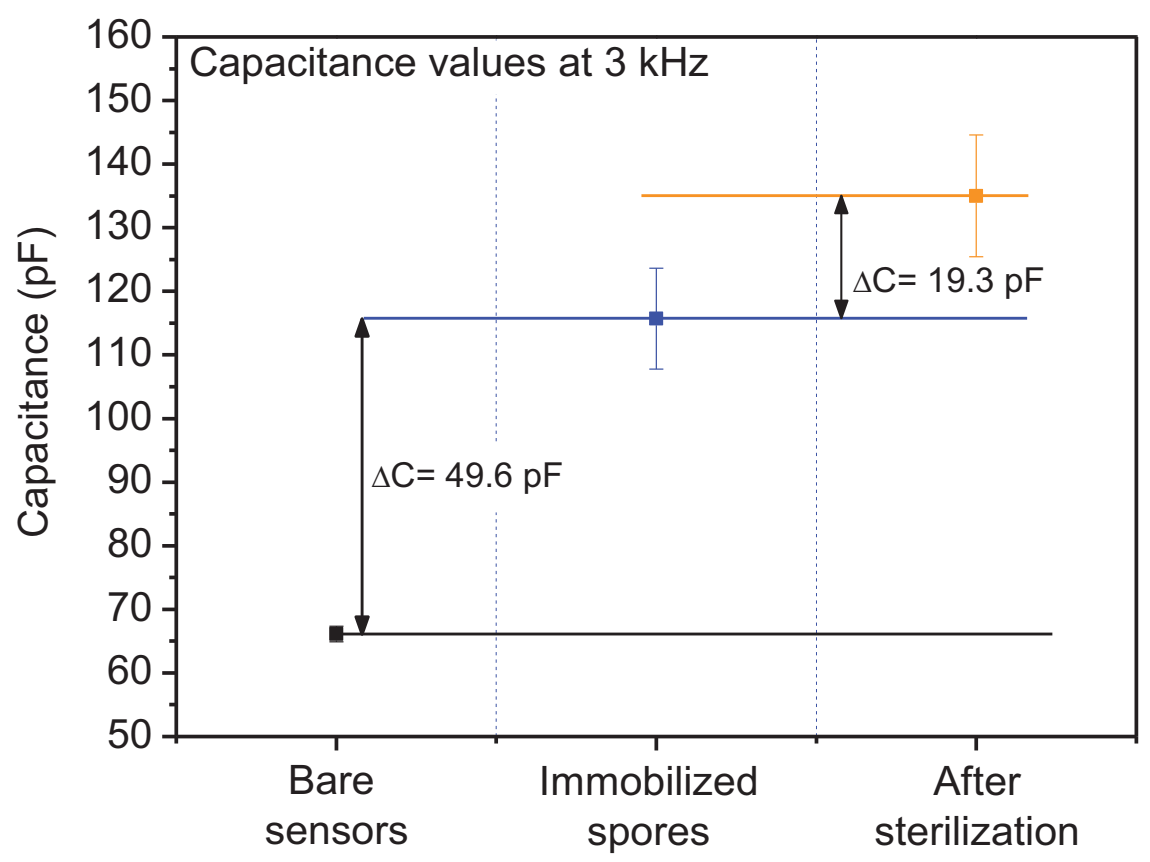

Fig. 5: Resulting capacitance values of the three different states at $3 \mathrm{kHz}$, calculated from data of Fig. 4 and application of eq (4). 


\section{Results of numerical validation}

The 2D-periodic FEM model was simulated for the case of a bare IDE structure. In order to analyze the spore layer, a porous medium was introduced over the bare structure, with a height of $500 \mathrm{~nm}$ corresponding to the spore diameter. The displacement field distribution for both cases is shown in Fig. 6.

By means of the FEM models, the capacitance of the bare structure was calculated to $60 \mathrm{pF}$. The relative error was $3.3 \%$ between analytical and numerical analysis. The relative error between numerical and experimental findings was $9.1 \%$. As such, the numerical and analytical errors compared to the experimental findings are matched. Hence, the difference between experimental and theoretical analysis can be related to the previously stated variations of the experiments (referring to the previous section).

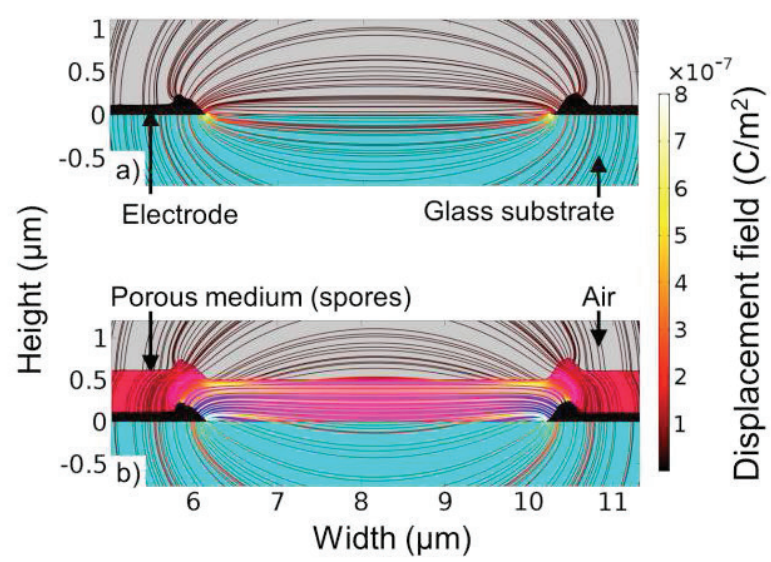

Fig. 6: FEM validations of the sensor structure. a) Bare IDE structure; b) IDE structure loaded with a porous medium representing the spore layer.

\section{Conclusions}

In the present work, an IDE-based sensor to monitor the efficiency of sterilization processes was presented. The sterilization process causes an alteration on the microbiological spore layer, which could be quantified as a capacitance change of about $19 \mathrm{pF}$.

Furthermore, an analytical expression was applied to verify the capacitance of the bare IDE structure. A relative error of $9.1 \%$ between measured and calculated value was noted.

In addition, experimental results were validated by means of an FEM-based model. The findings of the simulations were compared with the analytical verification and a small relative error of $3.3 \%$ was determined. Finally, the experimental results and the FEM model were combined to enable a derivation of the electrical properties of the porous spore layer.

\section{References}

[1] MCDONNELL, G.: The use of hydrogen peroxide for disinfection and sterilization applications. In: PATAI'S Chemistry of Functional Groups: John Wiley \& Sons, Ltd, 2014

[2] VDMA: Code of practice-filling machines of VDMA hygiene class $\mathrm{V}$ : testing the effectiveness of packaging sterilization devices. Vol. 6. Frankfurt am Main, Germany: Verband Deutscher Maschinen- und Anlagenbau e. V., 2008

[3] GERWEN, P. V. ; LAUREYN, W. ; LAUREYS, W. HUYBERECHTS, G. ; BEECK, M. O. D. ; BAERT, K. ; SULS, J. ; SANSEN, W. ; JACOBS, P. ; et al.: Nanoscaled interdigitated electrode arrays for biochemical sensors. In: Sens. Actuators B Vol. 49 (1998), Issue 1-2, pp. 73-80

[4] KITSARA, M. ; GOUSTOURIDIS, D. ; CHATZANDROULIS, S. ; CHATZICHRISTIDI, M. ; RAPTIS, I. ; GANETSOS, T. ; IGREJA, R. ; DIAS, C.: Single chip interdigitated electrode capacitive chemical sensor arrays. In: Sens. Actuators $B$ Vol. 127 (2007), Issue 1, pp. 186-192

[5] LAUREYN, W. ; NELIS, D. ; VAN GERWEN, P. ; BAERT, K. ; HERMANS, L. ; MAGNEE, R. ; PIREAUX, J.-J.; MAES, G.: Nanoscaled interdigitated titanium electrodes for impedimetric biosensing. In: Sens. Actuators B Vol. 68 (2000), Issue 1, pp. 360-370

[6] OBERLÄNDER, J.; BROMM, A. ; WENDELER, L. ; IKEN, H. ; PALOMAR DURÁN, M. ; GREEFF, A. ; KIRCHNER, P. ; KEUSGEN, M. ; SCHÖNING, M. J.: Towards a biosensor to monitor the sterilization efficiency of aseptic filling machines. In: Phys. Status Solidi A Vol. 212 (2015), Issue 6, pp. 1299-1305

[7] KIRCHNER, P. ; NG, Y. A. ; SPELTHAHN, H. ; SCHNEIDER, A. ; HENKEL, H. ; FRIEDRICH, P. ; KOLSTAD, J. ; BERGER, J. ; KEUSGEN, M. ; et al.: Gas sensor investigation based on a catalytically activated thin-film thermopile for $\mathrm{H}_{2} \mathrm{O}_{2}$ detection. In: Phys. Status Solidi A Vol. 207 (2010), Issue 4, pp. 787-792

[8] KIRCHNER, P. ; OBERLÄNDER, J. ; SUSO, H.P. ; RYSSTAD, G. ; KEUSGEN, M. ; SCHÖNING, M. J.: Monitoring the microbicidal effectiveness of gaseous hydrogen peroxide in sterilization processes by means of a calorimetric gas sensor. In: Food Control Vol. 31 (2013), Issue 2, pp. 530538

[9] ABU-ABED, A. S. ; LINDQUIST, R. G.: Capacitive interdigital sensor with inhomogeneous nematic liquid crystal film. In: Prog. Electromagn. Res. B Vol. 7 (2008), pp. 75-87

[10] LVOVICH, V. F. ; LIU, C. ; SMIECHOWSKI, M. F.: Optimization and fabrication of planar interdigitated impedance sensors for highly resistive nonaqueous industrial fluids. In: Sens. Actuators $B$ Vol. 119 (2006), Issue 2, pp. 490-496

[11] OLTHUIS, W. ; STREEKSTRA, W. ; BERGVELD, $P .:$ Theoretical and experimental determination of cell constants of planar-interdigitated electrolyte conductivity sensors. In: Sens. Actuators B Vol. 24 (1995), Issue 1, pp. 252-256 
[12] CORTEZZO, D. ; KOZIOL-DUBE, K. ; SETLOW, B. ; SETLOW, P.: Treatment with oxidizing agents damages the inner membrane of spores of Bacillus subtilis and sensitizes spores to subsequent stress. In: J. Appl. Microbiol. Vol. 97 (2004), Issue 4, pp. 838-852

\section{Acknowledgement}

This work has been financially supported by the Federal Ministry of Education and Research (BMBF), Germany, Project: "ImpediPack". 